\title{
A High Spectral Resolution Lidar Designed for Unattended Operation in the Arctic.
}

\author{
E. W. Eloranta and P. Ponsardin \\ University of Wisconsin-Madison \\ eloranta@lidar.ssec.wisc.edu
}

The University of Wisconsin High Spectral Resolution Lidar (HSRL) provides vertical profiles of optical depth, backscatter cross section, depolarization and backscatter phase function. All HSRL measurements are absolutely calibrated by reference to molecular scattering which is measured at each point in the lidar profile (Grund and Eloranta 1991, Pirronen and Eloranta 1994). This enables the HSRL to measure backscatter cross section and optical depth without a prior assumptions about the scattering properties of the atmosphere. Rigorous error estimates can be computed for all measurements.

This paper describes the design and initial testing of a new HSRL for long term unattended observations of arctic clouds and hazes. These observations are needed to improve the parameterizations of radiative transfer in global climate models.

The current HSRL requires attention by a highly trained operator and is not suited for long term measurement campaigns. Extensive modifications are required to fit this semi-trailer sized system into a package about the size of a phone booth and to allow operation and data transfer to be controlled remotely over the Internet.

One of the most troublesome features of the current HSRL involves maintaining alignment of the laser beam with the receiver field of view. Alignment is sensitive to perturbations at the $10 \mu \mathrm{rad}$ level. This problem will be avoided by employing a transceiver configuration where the transmitter and receiver share the same $0.4 \mathrm{~m}$ diameter telescope ( Fig. 1). In addition, the large transmitter aperture will limit both the per shot energy density and the multi-pulse average power density to eye-safe levels at the exit port. A passive polarization transmit-receive (T-R) switch consisting of a polarization beam splitter and a 1/4-wave plate will be employed. Our first critical test will be to measure the parasitic light incident on the detectors during emission of the laser pulse. The combined rejection of the polarization T-R switch and the $\mathrm{I}_{2}$ filter on-line attenuation is expected to protect the molecular channel detector from overload. However, light levels at the combined channel PMT and the cross-polarization channel APD may require additional blocking with a shutter. A rotating mechanical shutter capable of opening within 1 microsecond of the laser pulse has been designed to provide this additional isolation if it is required. 


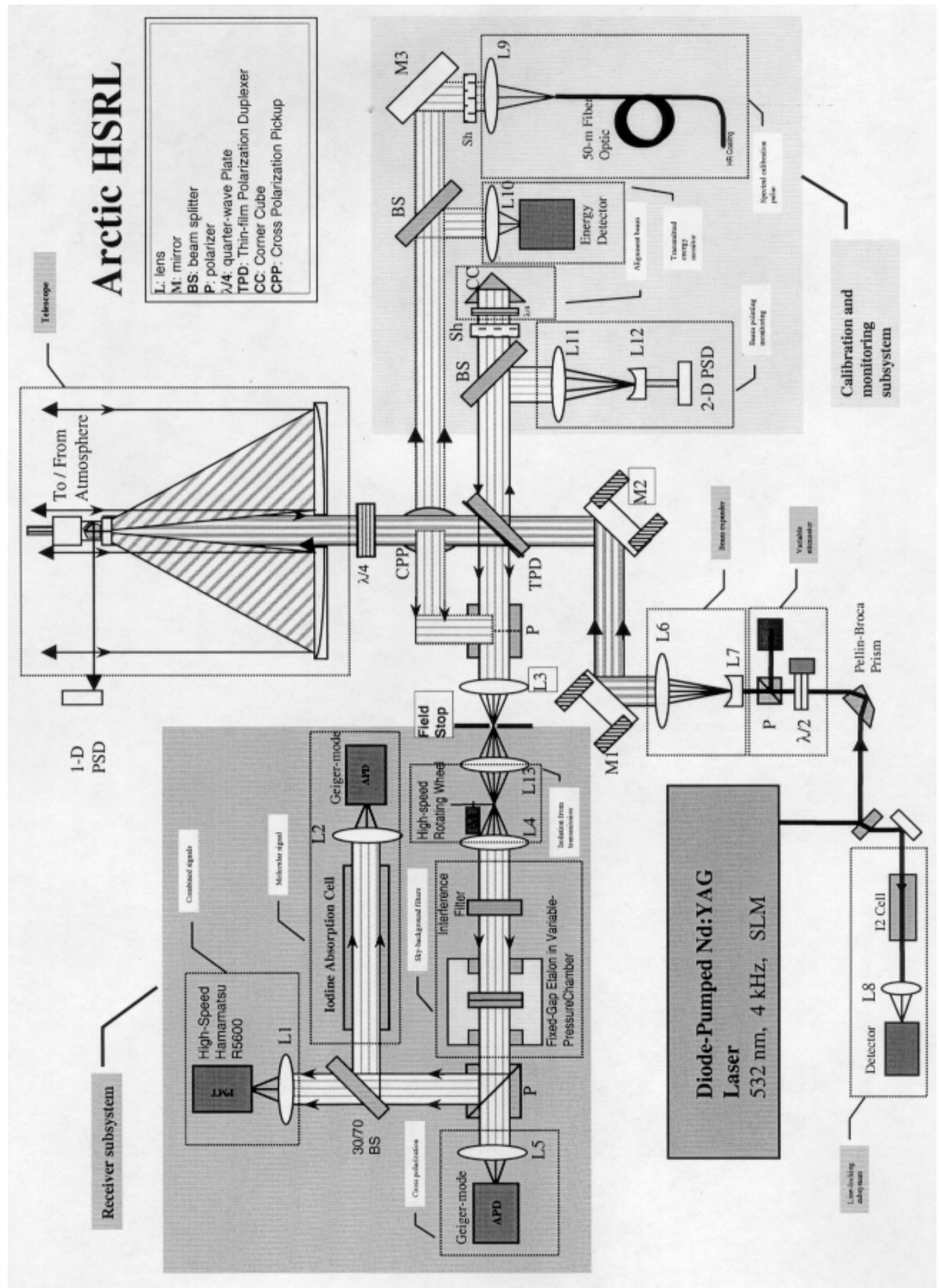

Figure 1: 


\section{Transmitter}

Average power

Spectral width

Pulse repetition rate

Beam divergence

Aperture

Aperture

Angular field of view

Spectral bandpass

$\mathrm{I}_{2}$ Blocking filter bandwidth

Molecular channel detector

Combined molecular/aerosol channel detector

Crossed polarization channel detector
$0.6 \mathrm{~W}$

$<0.1 \mathrm{pm}$

$4 \mathrm{kHz}$

$<50 \mu \mathrm{rad}$

$0.4 \mathrm{~m}$

Receiver

$0.4 \mathrm{~m}$

$100 \mu \mathrm{rad}$

$8 \mathrm{pm}$

$1.8 \mathrm{pm}$

EG\&G Geiger mode APD

Hamamatsu 7400 PMT

EG\&G Geiger mode APD

Table 1: Arctic HSRL Specifications

The etalon filter used in the current HSRL employs a flow through $\mathrm{N}_{2}$ pressure regulation system for tuning which is not suitable for unattended operation. We have constructed a closed system where pressure tuning of the etalon is accomplished using a stepper-motor driven bellows.

Arctic locations report a large number of days with very low cloud ceilings. This is a problem for our narrow field-of-view lidar design which does not collect light from the entire transmitter illuminated area until the laser pulse is $\sim 3 \mathrm{~km}$ from the lidar. Incomplete overlap does not effect backscatter cross section and polarization measurements because these quantities are are derived from ratios of signals. However absolute measurements of the molecular return are required to compute optical depth. The overlap function required for this measurement will be obtained using a computer controlled focus adjustment on the telescope secondary mirror. A calibration sequence performed periodically during routine operation will observe the molecular lidar return while scanning the telescope focus to map the overlap function.

The design of the arctic HSRL is complete. Major components have been ordered with delivery expected shortly. Assembly and testing of the testing should be underway prior to this meeting. This paper will provide a detailed description of the design and results of initial testing.

\section{References}

- Grund, C. J. and E. W. Eloranta, 1991: The University of Wisconsin High Spectral Resolution Lidar, Optical Engineering, 30, 6-12.

- Piironen, P. and E. W. Eloranta, 1994: Demonstration of a high-spectral-resolution lidar based on an iodine absorption filter. Optics Letters, 19, 234-236. 